\title{
XIV. Zur Funkenentladung des Inductoriums; Entgegnung auf die Bemerkungen des Hrn. von Oettingen $\left.{ }^{1}\right)$; von Adolf Heydweiller.
}

Hr. v. Oettingen beschwert sich, dass ich in meiner vorjährigen Arbeit über Funkenentladungen des Inductoriums ${ }^{2}$ ) seine diesbezüglichen Messungen nicht erwähnt habe. Die Arbeiten des Hrn. v. Oettingen über Funkenentladungen behandeln vorzugsweise Leydener Flaschen, die ich einer besonderen Betrachtung vorbehalten hatte. Seither hatte ich Veranlassung, mich eingehend mit denselben zu beschäftigen und werde demnächst auf sie zurückkommen. Dass Hr. v. Oettingen gelegentlich auch einige galvanometrische Messungen am Inductorium ohne Anwendung von Condensatoren vorgenommen, war mir leider entgangen; ich bedaure das, bezweifle aber, dass eine frühere Kenntniss dieser Messungen von Einfluss auf meine Arbeit gewesen wäre; denn Hr. v. Oettingen hat die Aufgabe, welche ich mir hauptsächlich gestellt, nicht berührt, die Aufgabe nämlich, eine Beziehung zwischen den entladenen Electricitätsmengen und den wirkenden electromotorischen Kräften zu finden, die über das Verhalten von Funkenstrecken beim Electricitätsdurchgang Aufschluss gäbe in ähnlicher Weise, wi $\theta$ das Verhalten von Leitungswiderständen durch das $\mathrm{Oh}$ m'sche Gesetz festgestellt ist. $\mathrm{Zu}$ diesem $\mathrm{Z}$ wecke habe ich die in Fig. 5 der genannten Arbeit graphisch dargestellten Reihen beobachtet. $\mathrm{Hr}$. v. Oettingen dagegen hat aus jeder Reihe je einen auf

1) A. J. v. Oettingen, Wied. Ann. 40. p. 74. 1890.

2) Heydweiller, Wied. Ann. 38. p. 534. 1889. In dieser Abhandlung sind zwei Druckfehler zu berichtigen: p. 535 , Zeile 9 v. u. ist zu lesen: $5000000 \mathrm{~cm}$ statt $500000 \mathrm{~cm}$ und p. 552 , Zeile 13 จ. $0 . b=B / w_{0}$ statt $c=B / w_{0}$. 
derselben Ordinate liegenden Werth bestimmt und ist dann allerdings zu grösseren Funkenstrecken fortgeschritten; eine primäre Stromstärke entsprach etwa der grössten von mir angewandten, wie ich mangels anderer Angaben aus dem $V$ erhältniss der gemessenen Electricitätsmengen bei den Funkenstrecken 0 und $0,05 \mathrm{~cm}$ schliesse, da sein Inductorium von nahe gleicher Grösse, wie das meinige war. Um für die grossen Funkenstrecken (bis zu 3,4 cm) Reihen, wie die von mir bestimmten zu erhalten, hätte ich zu wesentlich grösseren primären Stromstärken übergehen müssen, und diesen wollte ich meine neu hergestellten Gralvanometerrollen, die noch zu vielen Messungen dienen sollten, nicht aussetzen, nachdem die älteren Rollen der Wiedemann'schen Bussole trotz der dicken Guttaperchaisolirung sich für dieselben als unzuverlässig erwiesen hatten, zumal ich von einer weiteren Ausdehnung der Versuche keine wesentlich neuen Ergebnisse erwarte.

Ob Hrn. v. Oettingen's Galvanometer für so hohe Spannungen noch ausreichend gewesen wäre, ist aus seinen Arbeiten nicht ersichtlich; da auch seine Empfindlichkeit erheblich geringer, als die des meinigen war, so bedürfte es wohl noch eines genaueren Nachweises der von Hrn. v. Oettingen behaupteten grösseren Leistungsfähigkeit seiner Messvorrichtung.

Auch die übrigen Ausstellungen, die Hr. v. Oettingen an meiner Arbeit zu machen hat, sind durchweg nicht zutreffend. Nur das will ich gerne zugeben, dass in meinem Satze, „dass bei Apparaten von so grossen Dimensionen etc. die Bedingungen zur Entstehung von Schwingungen nicht günstig sind,“ (p. 541) an Stelle von ,Schwingungen“ schwingende Entladungen stehen sollte, denn das war in der That meine Meinung.

Bei der Herstellung der schematischen Fig. 6 war ich von der Anschauung ausgegangen, dass die starken Potentialänderungen in dem leitenden Theile des secundären Kreises, welche das schnelle Anwachsen der Stromintensität bedingen, das Eintreten der Entladung begünstigen, und dass dieser Einfluss vielleicht die Herabsetzung der Entladungsintensität durch die Luftrerdünnung in der Funkenstrecke übersteige; 
indessen mag ich mich hierin irren. Genügende Anhaltspunkte zur Beurtheilung dieser Frage sind meines $W$ issens noch nicht vorhanden und auch in den Arbeiten des Hrn. v. Oettingen nicht enthalten.

Hr. v. Oetting en wirft mir vor, dass meine Anschauungen nicht neu seien, sondern bereits vor 30 Jahren gegolten hätten. Dabei steht in seiner 1863 veröffentlichten Abhandlung $\left.{ }^{1}\right)$ : „Nachdem die Luftstrecke einmal durchbrochen ist, wird der gebildete Funkenkanal, der Erfahrung gemäss die Electricität ähnlich, wie ein metallischer Draht leiten (wir haben wenigstens noch kein Mittel, uns eine andere Vorstellung zu verschaffen)" - und spricht auch jetzt noch ${ }^{2}$ ) von einem "Widerstand" der Luftstrecke, während ich mich (p. 548 u. 549) auf das Deutlichste und Bestimmteste gegen diese Vorstellung ausgesprochen und an ihrer Stelle (p. 549) eine andere einzuführen versucht habe. - Ich legte den grössten Werth darauf, mich von den. Besonderheiten meines Apparates unabhängig zu machen, indem ich an Stelle der primären Stromstärken die Zeitintegrale der wirksamen electromotorischen Kräfte einführte, und Hr. v. Oettingen behauptet, ,ich habe dieselben nur zur Ermittelung der Abscissenwerthe verwendet und ihnen weiter keinen theoretischen Werth zugesprochen." - Ich suche auf allerdings umständlichem experimentellem Wege eine Beziehung zwischen den im Funken entladenen Electricitätsmengen und den Zeitintegralen der wirksamen electromotorischen Kräfte abzuleiten, und Hr. v. Oettingen nennt diese empirische Beziehung „eine Formel auf rationeller Grundlage“, hätte sie auch seinerseits viel einfacher abgeleitet, indem er die strömende Menge einfach direct proportional dem um das Entladungspotential verminderten vollen electromotorischen $\mathrm{Kraft}$ integral, und dem Gesammtwiderstande umgekehrt proportional gesetzt hätte, und ferner den "Widerstand der Luftstrecke" umgekehrt proportional jenem Kraftintegral, welche umgekehrte Proportionalität ihm "wohlbegründet" erscheint. Zunächst ist mir unklar, woher Hr. v. Oettingen diese Be-

1) v. Oettingen, Pogg. Ann. 118. p. 379. 1863.

2) v. Oettingen, Wied. Ann. 40. p. 81. 1890. 
gründung nehmen will, denn dass der „Widerstand der Luftstrecke" ihrer Erwärmung durch den Inductionsfunken umgekehrt proportional und diese dem electromotorischen Kraftintegral direct proportional sei, ist doch wohl noch nicht nachgewiesen. Ferner ist die Grösse $B$ in meiner Formel $q=\left(q_{0} \omega_{0}-B\right) /\left(\omega+C / q_{0} \omega_{0}\right)$ durchaus nicht das Entladungspotential; vielmehr sieht man leicht, dass sie dieselbe Dimension, wie $q_{0} \omega_{0}$, d. h. eines Zeitintegrales einer electromotorischen Kraft hat; es ist daher auch nicht zutreffend, dass ich sie, wie Hr. v. Oettingen behauptet, "sehr passend Entladungspotential" genannt habe; vielmehr ist diese Bezeichnung durchaus nicht passend. Die physikalische Bedeutung von $B$ habe ich $^{1}$ ) ausführlich erörtert. - Ich hatte den möglichen Fehler bei der Bestimmung der Grösse $C$ der vorstehenden Formel aus den Beobachitungen auf 5 bis 10 Proc. angegeben; Hr. v. Oettingen scheint hierin einen Widerspruch mit der Thatsache zu finden, dass die Formel bei starker Aenderung von $C$ nicht mehr den Beobachtungen genügt, übersieht aber dabei, dass zwei Grössen $B$ und $C$ gleichzeitig aus den Beobachtungen abzuleiten sind, und dass man bei entsprechender Aenderung beider Grössen die Beobachtungen noch sehr wohl durch die Formel darstellen kann.

Eine letzte Bemerkung des Hrn. v. Oettingen richtet sich gegen meinen Versuch, die Zeitdauer der Electricitätsbewegung durch zugeschaltete electromotorische Kräfte von bekannter Grösse zu bestimmen, und empfiehlt dagegen die Methode des rotirenden Spiegels. Aber die letztere gestattet nur die Zeitdauer der optischen, nicht der electrischen Erscheinung zu bestimmen, und es ist in jedem Fall zu untersuchen, wie weit beide zusammenfallen. Schon Feddersen, dem jene Methode ihre vorzüglichste Ausbildung verdankt, weist darauf hin, dass dies nicht immer der Fall. ${ }^{2}$ ) Die Einschaltung von Geissler'schen Röhren in den Entladungskreis führt ausserdem neue, nicht genügend bekannte Verhältnisse in die Versuchsanordnung ein.

1) A. Heydweiller, l. c. p. 549.

2) Feddersen, Pogg. Ann. 113. p. 437. 1861. 
Auf eine nochmalige genauere Darlegung meiner Anschauungen über die Wirkung von Funkenstrecken auf die Electricitätsbewegung an dieser Stelle verzichte ich, da ich auf dieselben demnächst an der Hand neuen Beobachtungsmaterials ausführlicher einzugehen und dabei den Nachweis zu versuchen gedenke, dass die Annahme einer electromotorischen Gegenkraft so wenig, wie die eines Widerstandes im Funken, mit den Thatsachen in Uebereinstimmung ist.

Wurzburg, im Mai 1890. 